Artículo de reflexión

Cómo citar: Goyes, G. (2020). El valor social de la comunicación visual. Reflexiones educativas de una Mediaciones, 25 (16). Pp. 348-361. mediaciones.16.25.2020.348-361

Editorial: Corporación Universitaria Minuto de Dios - UNIMINUTO

Recibido: 7 de septiembre de 2020 Aceptado: 16 de octubre de 2020 Publicado: 16 de diciembre de 2020

ISSN: 1692-5688 | eISSN: 2590-8057 formación disciplinar emergente. En: https://doi.org/10.26620/uniminuto.

\section{El valor social de la comunicación visual. Reflexiones educativas de una formación disciplinar emergente.}

\section{The social value of Visual Communication. Educational reflections of an emerging discipline}

\section{0 valor social da Comunicação Visual. Reflexões educacionais de uma formação disciplinar emergente}

\section{Gilberto Goyes}

ggoyes@auniminuto.edu

https://orcid.org/0000-0001-6354-728X Administrador de empresas

Universidad Minuto de Dios UNIMINUTO

Colombia
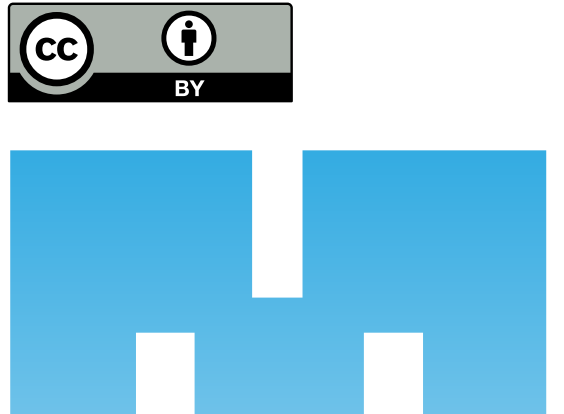

\section{Resumen}

Hablar de comunicación visual hace alusión a la imagen y lo visual, a la relación entre imagen y cultura; una relación que posibilita construcciones simbólicas en torno a experiencias, condiciones de vida, identidades, modos de habitar un territorio e imaginarios. Esto posibilita otras formas de construcción de sentido y significado que se sustentan a partir de la relación comunicación-imagencultura.

"El mundo-imagen es la superficie de la globalización. Es nuestro mundo compartido. Empobrecida, oscura, superficial, esta imagen-superficie es toda nuestra experiencia compartida. No compartimos el mundo de otro modo. El objetivo no es alcanzar lo que está bajo la superficie de imagen: sino ampliarla, enriquecerla, darle definición, tiempo. En este punto emerge una nueva cultura”. (Buck-Morss, 2009, pág. 42).

Es relevante comprender y diferenciar qué significa y no significa la comunicación visual; esto permite determinar diferencias conceptuales para hallar elementos que den mayor pertinencia al subcampo y la disciplina.

Palabras clave: Comunicación, comunicación visual, imagen, cultura visual, imaginarios, diseño, lo visual, visualidad. 


\section{Abstract}

Talking about visual communication refers to the image and the visual, to the relationship between image and culture; a relationship that enables symbolic constructions around experiences, living conditions, identities, ways of inhabiting a territory and imaginary. This makes possible other forms of construction of meaning and meaning that are sustained from the communication-image-culture relationship.

Estas reflexiones son derivadas del proceso de creación y puesta en marcha del programa profesional en Comunicación Visual de la Facultad de Ciencias de la Comunicación de Uniminuto Bogotá, Sede Principal.

El autor ha declarado que no existen intereses en competencia.

"The world-image is the surface of globalization. It is our shared world. Impoverished, dark, superficial, this surface-image is our entire shared experience. We do not share the world in any other way. The objective is not to reach what is under the image surface: but to enlarge it, enrich it, give it definition, time. At this point a new culture emerges ”. (Buck-Morss, 2009).

It is relevant to understand and differentiate what visual communication means and does not mean, this allows determining conceptual differences to find elements that give greater relevance to the sub-field and the discipline.

Keywords: Communication, visual communication, image, visual culture, imaginaries, design, the visual, visuality.

\section{Resumo}

Falar de comunicação visual refere-se à imagem e ao visual, à relação entre imagem e cultura; uma relação que possibilita construções simbólicas em torno de experiências, condições de vida, identidades, modos de habitar um território e imaginário. Isso possibilita outras formas de construção de sentido e sentido que se sustentam na relação comunicação-imagem-cultura.

"A imagem mundial é a superfície da globalização. É nosso mundo compartilhado. Empobrecida, escura, superficial, essa imagem superficial é toda a nossa experiência compartilhada. Não compartilhamos o mundo de nenhuma outra forma. O objetivo não é atingir o que está sob a superfície da imagem, mas ampliá-la, enriquecê-la, dar-lhe definição, tempo. Nesse ponto surge uma nova cultura ”. (Buck-Morss, 2009).

É relevante compreender e diferenciar o que significa comunicação visual e o que não significa, pois permite determinar diferenças conceituais para encontrar elementos que dêem maior relevância ao subcampo e à disciplina.

Palavras chave: Comunicação, comunicação visual, imagem, cultura visual, imaginários, design, o visual, visualidade. 


\section{Sobre el diseño gráfico, la comunicación y la comunicación visual}

Para comprender la conceptualización de comunicación visual se identificaron tres escenarios: El primero, reconocer la naturaleza de las disciplinas afines a la comunicación y al diseño; el segundo, a partir de la comprensión de la naturaleza misma de la comunicación visual a través de un proceso de decantación de cada uno de los conceptos que la constituyen; y el tercero, amplía el objeto de estudio de la comunicación visual, desde la comprensión de elementos transversales de la Comunicación Visual como disciplina.

Como marco de referencia iniciamos con lo expuesto por Brea respecto al poder significacional de lo visual en la cultura:

Si a ello se añade la fabulosa potenciación de su papel - el de la cultura visual - en las sociedades contemporáneas, debida a las posibilidades ofrecidas por las nuevas tecnologías (no sólo de cara a su producción, sino sobre todo de cara a multiplicar hasta límites inimaginables sus potenciales de distribución y por tanto de condicionamiento de modos de vida), podemos con seguridad afirmar que nos enfrentamos frente a un horizonte de transformaciones importantísimas en cuanto al campo de las prácticas productoras de significado cultural por medio de la visualidad (así como para las disciplinas que pretenden su estudio e interpretación). (Brea, 2003, pág. 5).

\subsection{Primer escenario: enfoques y posturas de la comunicación visual}

Parte de un análisis con diferentes posturas frente al tema de la comunicación y de la imagen, desde dos enfoques, uno entendido desde una relación objeto-arte-diseño y el cual tiene una aproximación al diseño gráfico; y otro, que está más dado a la relación mensajes-códigos, propio al campo de la comunicación. Dichos enfoques, permiten visibilizar una relación ineludible, simbiótica entre comunicación y diseño gráfico. Esta relación constituye una de las posturas desde las cuales se puede entender la comunicación visual como un punto de convergencia de dichas aproximaciones académicas y conceptuales. Desde esta perspectiva, se vienen desarrollando diferentes programas académicos en Colombia.

En el entramado de la comunicación visual entre dos disciplinas, la comunicación y el diseño gráfico son dos fenómenos sumamente complejos incapaces de ser restrictivos o subordinados uno al otro; porque la comunicación no es una función exclusiva del diseño gráfico, y éste no surgió solamente para satisfacer las demandas comunicacionales. Hablar de comunicación dentro del entramado cultural es hablar de ella como medio para entablar vínculos entre los miembros de una misma cultura o de diferentes culturas. Baldwin y Roberts dicen al respecto: "el diseño es una forma de comunicación; y la comunicación es la base de nuestras relaciones y de nuestra forma de comprender el mundo. [...] A su vez, es un elemento que determina la cohesión social de un grupo.” (Estupiñán Villanueva, Solano Andrade, \& Torres Hidalgo, 2014, pág. 118). 
Lo anterior, evidencia la necesidad de visibilizar las particularidades de disciplinas como diseño gráfico, diseño visual y comunicación gráfica, de manera tal que la propuesta disciplinar de Comunicación Visual tenga en cuenta esas particularidades, a fin de desarrollar un objeto de estudio que comprenda, desde aspectos más amplios la relación comunicación-imagen-cultura.

El diseño gráfico trabaja desde la postura objeto-arte-diseño, lo que significa que aborda la relación forma-contenido desde una relación simbólica y una abstracción del código en grafismos, para lograr unas formas visuales que faciliten la comprensión del mensaje.

El Diseño Gráfico se enfoca más en la parte operativa, instrumental, la esencia se encuentra en la percepción que determinará la detectabilidad y discriminabilidad de los mensajes específicamente de los estímulos visuales, los cuales tendrán mayor o menor poder de atracción dependiendo de la eficacia de las funciones del lenguaje visual que a su vez está determinado por ciertos códigos. (Estupiñán Villanueva, Solano Andrade, \& Torres Hidalgo, 2014, pág. 119).

Por otro lado, el diseño visual "es un área profesional del diseño que sistematiza los códigos visuales en mensajes, según los principios formales y conceptuales del diseño, los modos de la comunicación y los tipos en los que aparecen los significantes de los signos icónicos" (Universidad de Caldas, 2020), por lo tanto se enmarca de acuerdo a lo establecido por el Ministerio de Educación Nacional de Colombia: "La creación, estructuración y ordenamiento de códigos, lenguajes y medios que permitan la legibilidad e interpretación de datos y mensajes gráficos, visuales y multimedia. Dentro de este campo disciplinario el programa podrá tener una de las siguientes denominaciones académicas: Diseño Gráfico, Diseño Visual.” (Ministerio de Educación Nacional de Colombia, 2003, pág. 2).

Como denominación profesional del diseño visual determina la pertinencia de los soportes de la imagen, teniendo en cuenta la naturaleza de la información para categorizar las formas visuales que ayudarían a la ejecución general de un proyecto. El diseño visual estudia la producción de la imagen a fin de comprender los grados de interactividad en los dispositivos y comprender el objeto como mediador de la relación sujeto-contenido.

De otra parte, la comunicación gráfica se sustentan desde la relación mensaje-formaaudiencia, posturas más cercanas como evolución de las artes gráficas. Esta se centra en el estudio de la utilización de forma persuasiva de artefactos gráficos para la transmisión de mensajes, el intercambio de sentidos y la puesta en común de significados, todo esto mediado inicialmente por los medios impresos (grafías) y posteriormente integrando las TIC. El objeto de estudio dela comunicación gráfica publicitaria, "Lo constituye la comunicación persuasiva, entendida como el intercambio de sentidos inherente a los procesos comunicativos de ideas, bienes y servicios, desde una perspectiva estratégica, donde los lenguajes estéticos responden a la dinámica de lo persuasivo en el contexto sociocultural. Se asume la comunicación como intercambio de sentidos, como las interacciones y transacciones humanas que permiten la puesta en común y la comprensión a través de lenguajes y que de este modo, contribuyen a la construcción de cultura”. (Universidad de Medellín, 2020, pág. 19). 
Para lograr lo anterior, la comunicación gráfica se apoya en la relación interdisciplinar de la comunicación, el diseño y la publicidad. Esta relación triádica permite a esta disciplina, a partir de la detección de las realidades de las audiencias y las necesidades de los clientes, la construcción de artefactos gráficos (grafías) - impresos y digitales - que permitan de forma persuasiva y estratégica, la producción, la circulación, la puesta en común y el intercambio de sentidos, soportados en los medios y las TIC.

Así pues, la comunicación gráfica parte de comprender que la comunicación es un acto humano de interacción social por excelencia, y por ende es un componente fundamental para la compresión de la cultura, de allí que cualquier artefacto gráfico o grafía, sin importar su naturaleza, es en sí mismo un bien cultural simbólico; la publicidad, como una disciplina que permite comprender los públicos y las audiencias, y como de forma persuasiva incidir en éstas, con el fin de modificar comportamientos, establecer ideas o promover bienes y servicios; y el diseño gráfico, como la disciplina que permite la preformación de ideas en realidades comunicativas, para la solución de problemáticas dadas o existentes.

\subsection{Segundo escenario: reflexiones y naturaleza de la comunicación visual}

A partir de la revisión y reflexión de la naturaleza de la comunicación visual y los elementos conceptuales que intervienen dentro de su estructura de conocimiento en dos momentos: la aproximación a los conceptos de comunicación y la relación existente entre la imagen y lo visual.

El proceso de decantación y aproximación a los conceptos de comunicación y de lo visual, en donde dichas aproximaciones comprenden la comunicación como un campo transdisciplinar. Si bien esta tiene que ver con todas las dinámicas de interacción social, en donde el ser humano es constante productor, consumidor e intérprete de signos y sentidos, ella no puede desligarse de la cultura, de las experiencias, los aprendizajes y las relaciones sociales. Respecto a la comunicación como campo José Miguel Pereira afirma:

Los debates contemporáneos en América Latina perfilan a la Comunicación más como un campo de conocimiento transdisciplinario que como una disciplina. Es un campo de conocimiento desde el cual se puede comprender, interpretar e intervenir a múltiples niveles los procesos de interacción y significación a través de la creación, circulación y usos de medios y tecnología y de formas simbólicas con multiplicidad de perspectivas: social, cultural, ética, política, estética y económica, entre otras. (Pereira, 2005, pág. 421).

Entender la relación existente entre la imagen y lo visual, que está dada a partir de la representación y la significación. Estas a su vez generan procesos de simbolización que adquieren sentido desde la cultura, y permiten hablar del fenómeno de la cultura visual como un elemento de producción cultural-social de la imagen. 
Para alcanzar este objetivo, nuestro punto de partida ha sido pensar la imagen como producto y como proceso cultural. La presencia de las imágenes en nuestra vida cotidiana es tan importante y tan masiva que tendemos a considerarlas como parte de nuestro entorno "natural", como representación o reflejo de una realidad "externa”. El análisis cultural de la imagen nos recuerda que la imagen es un producto social, que su interpretación depende de convenciones arbitrarias y no sólo perceptivas, y que hemos aprendido a atribuirle sentido y significado a partir de patrones culturales muy elaborados, como pueden ser los formatos de las telenoticias, las series de ficción, la publicidad o los documentales. (Ardevol \& Muntañola, 2014, pág. 13).

Todo este desarrollo conceptual, propone abordar la comunicación visual no como el punto de encuentro entre variadas disciplinas, sino como un campo disciplinar y formativo que pretende interpretar las prácticas productoras de significado (puestas en escena, representaciones, eventos, expresiones, etc.) a través de lo visual.

Así pues, esta postura amplía las perspectivas de este campo de estudio, desde las relaciones de la imagen y lo visual, que permiten dimensionar el objeto de estudio de la comunicación visual hacia escenarios formativos de la imagen desde la comunicación, con implicaciones sociales, políticas y culturales, que establezcan una construcción del conocimiento dentro de las nuevas sociedades (comunicación - imagen - cultura).

Hay por ello una gran amplitud de dimensiones comprometidas desde las propiamente estéticas y críticas, a las de la función antropológica de la imagen en las sociedades actuales. (...) Desdela reflexión sobre la importancia de la visualidad en cuanto a la construcción identitaria -en los procesos de individualización y socialización- y de la sujeción, hasta la reflexión sobre el cambio del paradigma cultural y filosófico que podría conllevar un "giro" al desplazarse el centro de su ontología, desde el logos hasta la imagen, en la aparición inquietante y sugestiva de un nuevo pregnante "imagocentrismo". (Brea, 2003, pág. 6).

De esta manera, la imagen se asume como un elemento que es susceptible de estudio, dado que no solamente aborda aquello que es perceptible a través de la visión, sino que constituye una estructura teórico-conceptual que establece relaciones entre lo visual y la realidad, a partir de niveles de representación, significación-simbolización y creación, que se generan mediante procesos sintéticos.

La epistemología de la imagen se encuentra vinculada al campo del conocimiento y concretamente al campo del arte, como una forma de representar la realidad por medio de una discursividad constitutiva de la visualidad cultural y del saber de las ciencias por medio del lenguaje visual, al abordar el problema epistemológico fundamental de la concepción de la realidad, apropiándose de lo real, lo cognitivo, lo cultural y lo imaginario. (Finkel Ettingher, 2014, pág. 386). 
A diferencia de otras posturas, la disciplina de la Comunicación Visual asume la imagen y lo visual como categorías complementarias, dado que la imagen, al ser una estructura conceptual, se construye principalmente desde lo visual, entendiendo éste, como una de las formas y elementos para construir el conocimiento a través de la imagen.

[...] desde los planteamientos comunicativos de lo visual de Armando Silva, podemos comprender las propiedades de la imagen visual, sobre todo, porque lo verbal y lo no verbal quedan interrelacionados: nombrar es el correlato que destaca lo verbal, es decir que le da significado (por ejemplo: nombramos la "luna"); indicar es correlato de lo señalético en la medida en que al señalar la imagen (gesto paralingüístico) para acompañar la acción de nombrarla, hacemos un signo ostensivo; y mostrar es el correlato de lo visual o estético, puesto que la re-creación (de la luna en cuanto imagen) evita nombrarla, abriendo los caminos de la imaginación. Simbología, episteme y estética, nombrar, indicar y mostrar, no se dan aislados sino que configuran un tejido complejo que hace que estudiar la imagen sea impostergable hoy. (Goyes Narvaez, 2003, pág. 52).

\subsection{Tercer escenario: comunicación visual y lo visual}

En éste, se hace referencia a lo visual, concepto relativamente reciente que se sustenta desde la cultura visual y la interculturalidad, y que se entiende no sólo "como la construcción visual de lo social. Lo visual no se asocia a la percepción, sino a la construcción de lo social. Lo importante no es el ojo que mira (desde un punto de vista fisiológico) sino el campo social de la mirada, es decir, la construcción de la subjetividad, la identidad, el deseo, la memoria y la imaginación”. (AIG, 2020)

La Comunicación Visual toma como una de las bases epistemológicas, las relaciones transdisciplinares que existen entre la comunicación y los estudios visuales, y que permiten entender la imagen como un producto cultural, en un marco conceptual de campos en tensión.

La comunicación es un proceso de orden cultural y de contexto, que posibilita la mediación y la producción de sentido, y permite el reconocimiento del otro a partir de una interrelación mutua, donde tanto el individuo como el contexto se transforman. Dentro de este escenario de la comunicación también está presente la imagen, con sus propias dinámicas de circulación, consumo, construcción y producción, que en la actualidad, han permitido hablar del fenómeno de lo visual. Dicho fenómeno, se encarga del análisis desde los procesos de producción de significado cultural - cultura visual - y se aborda a partir de los estudios visuales, que permiten la interacción transdisciplinar: la historia del arte, la estética, la semiótica, la psicología, la cultura visual, la teoría de los medios y los estudios culturales, entre otras. Este enfoque permite que la cultura visual, conceptualmente hablando, encuentre como base epistemológica una mayor afinidad con la comunicación y el diseño. 
De esta manera, la convergencia transdisciplinar entre la comunicación y la cultura visual, genera un escenario propicio para el desarrollo de una disciplina profesional en comunicación visual, donde se posibilitan proyectos de comunicación, estudios y análisis de códigos visuales, diversos tipos de lenguajes que interactúan y se complementan, la imagen que atraviesa todos los ámbitos de la vida humana, los procesos de mediación tecnológica soportados en la imagen digital, nuevas formas y narrativas de imágenes envolventes, interactivas, virtuales, fijas y en movimiento, así como el análisis de los fenómenos relacionados con la construcción de conocimiento visual, el impacto cultural que éstos desencadenan, sus prácticas y aplicaciones en contextos sociales específicos, y la capacidad expresiva de los medios y las tecnologías.

En este marco de referencia, la Comunicación Visual se comprende como un campo transdisciplinar que abarca el estudio de la relación comunicación-imagen-cultura que se soporta en los procesos de creación, circulación, mediación y puesta en común de sentidos y significados compartidos que transcurren en los eventos visuales.

\section{Cambio de paradigma: del diseño gráfico a la comunicación visual}

Al revisar el contexto internacional del diseño gráfico, se encuentra el evento visual y la imagen como fundamento del mismo, es decir como estos son principalmente objeto y fin de los procesos comunicativos desde el origen de la humanidad y como se han mantenido y cobran cada vez más vigencia en la cultura actual, la cual es cada vez más mediada por la imagen. Se comprende entonces que el objeto de estudio del diseño gráfico se ha transformado y es cubierto por la sombrilla conceptual de la comunicación.

“[...] el diseño de comunicación visual, visto como actividad, es la acció de concebir, programar, proyectar y realizar comunicaciones visuales, producidas en general por medios industriales y destinadas a transmitir mensajes a grupos determinados. Esto se hace con el fin de afectar el conocimiento, las conductas o las actitudes de la gente en una dirección determinada. Un diseño gráfico es un objeto creado por esa actividad (Gorb, 1978, pág. 6). Esta actividad usa la evaluación sistemática de sus resultados como base para mejorar se eficiencia.” (Frascara, 2000, pág. 24).

Encontramos así como Dwiggins en 1922 acuña el término diseño gráfico para "describir sus actividades como individuo que aporta orden estructural y forma visual a las comunicaciones impresas” (Meggs, 1991, pág. 9) o la de Frascara: "El diseñador de comunicación visual trabaja en la interpretación, el ordenamiento y la presentación visual de mensajes” (Frascara, 2000, pág. 24). Mientras que Helmer se centra en el modelo funcionalista donde es profesional que simplemente es un escritor visual de mensajes:

El diseño gráfico es por lo tanto un proceso creativo que combina el arte y la tecnología para comunicar ideas. El diseñador trabaja con una variedad de herramientas de comunicación para transmitir un mensaje de un cliente a un 
público en particular. Las herramientas principales son la imagen y la tipografía. (Moreno Rodríguez, 2014, pág. 13).

Rollié y Branda afirman por su parte: “(...) la práctica del diseño requiere cada vez más de conocimientos amplios en los procesos de comunicación y en su conceptualización para poder laborar la complejidad estructural de los mensajes”. (Rollié \& Branda, 2004, pág. 35). Aquí es de suma importancia los postula Joan Costa en su artículo, Diseño de Comunicación Visual: el nuevo paradigma, donde plantea el cambio evolutivo que ha venido sufriendo la disciplina del diseño gráfico, para entrar en un encuentro directo con la comunicación. "Asumir la comunicación como función esencial del diseño gráfico, es salir fuera de la burbuja profesional para interactuar con las personas y la sociedad, a través del principal canal de percepción, de conocimiento y de recuerdo: el canal visual”. (Costa, 2014, pág. 96).

Este es un cambio evolutivo que parte de entender que los seres humanos están en constante relación e interacción con los demás, por lo tanto tienen la capacidad de producir, comprender y compartir mensajes e informaciones de todo tipo, en todo momento, lo cual no es ajeno a los procesos de comunicación visual. Ésta también hace parte de un proceso evolutivo que se nutre desde su fase más arcaica (el grafismo), mutando posteriormente a un sistema evolucionado, considerándolo una disciplina (diseño gráfico). Este último sienta sus bases formativas a partir de la escuela de la Bauhaus que considera como uno de sus principios fundamentales que la forma sigue la función. (Costa, 2014).

Esta evolución y su encuentro directo con la comunicación hace que viejas ideas funcionalistas sean superadas, primero con un punto de vista del ser humano como un ser visual, y segundo porque este interactúa directamente con el contexto, desde su percepción, conocimientos y recuerdos, todos engranados en un proceso sensorial de comunicación. Por consiguiente, el Comunicador Visual pasa a ser sujeto de cambio en donde con la comprensión que todo sistema, proyecto u objeto visual implican una manifestación cultural, y por ende circula, produce y pone en común significados, permite la construcción de sentidos comunes y propios, soportados en proceso de ideación, creación y producción de imágenes para transmitir mensajes, en donde la aproximación entonces se realiza primero desde la comunicación y luego desde el diseño.

Así pues, el estudio y comprensión del contexto propios de la comunicación dinamiza la capacidad de circulación, mediación y construcción colectiva de significados que transcurren en los eventos visuales y permite soluciones innovadoras, creativas, estéticas y estratégicas de problemáticas existentes en comunidades, entidades y organizaciones.

Este enfoque tiene una amplia relevancia dentro de los procesos sociales y culturales. Este cambio de definición del objeto de estudio del diseño gráfico se da globalmente y amplía la comprensión de los fenómenos que aborda llevándolos al "El diseñador capacitado profesionalmente aplica la intención de crear el entorno visual, material, 
espacial y digital, consciente de lo experiencial, empleando enfoques interdisciplinarios e híbridos de la teoría y la práctica del diseño.” (International Council of Design, 2020, pág. 23).

La apuesta epistemológica, metodológica, investigativa y formativa parte de una comprensión interdisciplinar y transdisciplinar de la comunicación visual, que desde la relación comunicación-imagen-cultura, permite el estudio, la creación y la gestión de sistemas y proyectos que promuevan la construcción de sentidos y significados compartidos que transcurren en los eventos visuales, y que posibilitan: ampliar la generación de conocimiento visual, mejorar las condiciones de relacionamiento humano, y comprender los fenómenos que encierran los procesos de comunicación desde la perspectiva de la imagen.

La comunicación visual debe permitir comprender y utilizar el poder y las potencialidades de la imagen en la solución de problemáticas sociales reales y concretas, apoyándose en una juiciosa investigación del contexto, soportado en los medios y las TIC disponibles en el repertorio tecnológico contemporáneo, y usando lenguajes textuales, visuales, orales, estéticos, interactivos, espaciales y de movimiento, todo esto con el objetivo de lograr la construcción de significados y sentidos compartidos, y en últimas el desarrollo a escala humana.

\section{Contexto y desarrollo desde la comunicación visual}

Contexto y desarrollo son escenarios dinámicos complementarios, pues el contexto no solo se refiere a la situación donde ocurren las cosas, sino que se constituye en el espacio en el cual la Comunicación Visual afecta a la sociedad, hace que las personas cambien o recreen sus referentes, sus prácticas y sus relaciones, se modifiquen los contextos, y en esa dinámica, se potencialicen procesos de desarrollo a escala humana.

Es en este escenario de contexto que se comprende que la responsabilidad social del Comunicador Visual está fundamentada en el marco de los principios humanistas, que reconozca el impacto o los grados de afectación que generan sus propuestas en diversos contextos, que promuevan la participación, el reconocimiento, la autonomía, la identidad, el consenso y la ciudadanía.

La Comunicación Visual entiende el desarrollo como un conjunto armónico del desarrollo de las personas y las comunidades (desarrollo humano y social), el desarrollo respetuoso de la naturaleza y los recursos (desarrollo sustentable), y del desarrollo que permita un beneficio económico sensato centrado en las personas y no solamente en los bienes materiales (desarrollo económico). 


\section{Figura 1}

\section{Concepción de desarrollo para la comunicación visual}

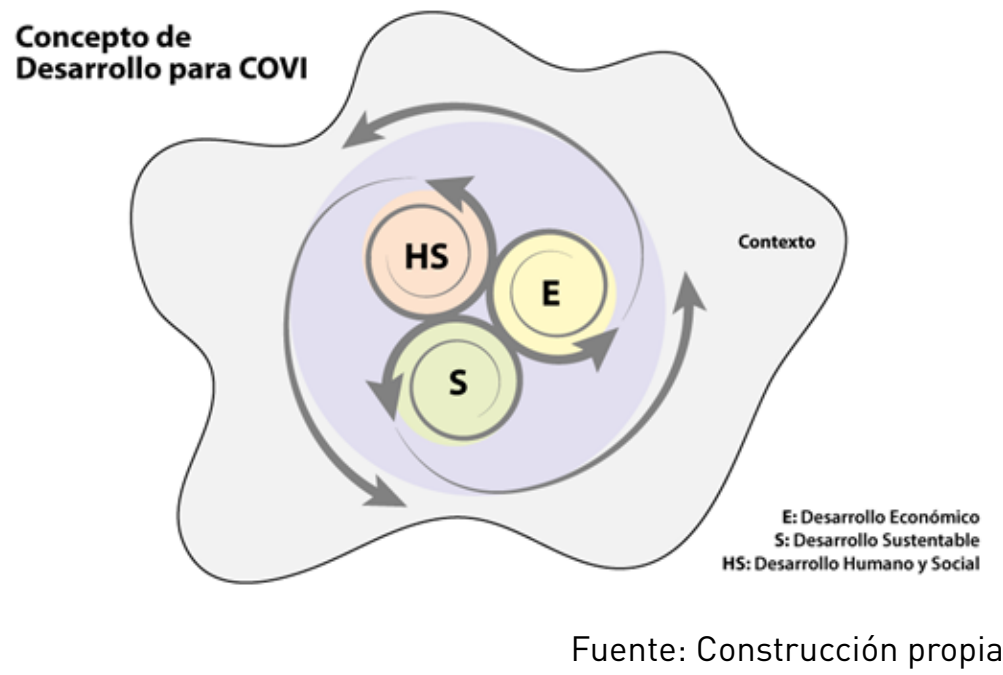

Desde esta óptica integral del concepto de desarrollo, puede abordarse a partir del desarrollo a escala humana, como lo diría Max Neef, "El desarrollo se refiere a las personas, no a los objetos". (Max-Neef, 1993). Así que transponiendo el postulado de Max Neef: la comunicación visual se refiere a las personas, no sólo al problema discursivo, ni a los medios, ni a los resultados. "Las necesidades humanas deben entenderse como un sistema en que las mismas se interrelacionan e interactúan” (Max-Neef, 1993, pág. 41).

Es así como este análisis de necesidades, relaciones y complejidades permite al Comunicador Visual, la construcción de sistemas visuales de comunicación que tengan en cuenta la mayor cantidad posible las variables, de forma tal que estos sean asertivos, eficientes, eficaces y adecuados a realidades específicas y concretas de las personas de las comunidades y organizaciones.

\section{El valor social del comunicador(a) visual}

En un continente atravesado por problemas complejos que resultan extensos y de difícil solución se requiere de Comunicadores Visuales que, por un lado, conozcan su territorio, su país, su continente, su historia, sus realidades y hagan parte de las transformaciones sociales y culturales que se requieran, con competencias en el actuar, el pensar y el ser, en lo social, en lo ético y en el entendimiento profundo de los contextos, que comprenda, juzgue, idee, ejecute y mida proyectos de comunicación visual que ayude a las personas a promover su desarrollo humano, ejercer la ciudadanía y la democracia participativa, el rescate de los bienes y los valores culturales de sus territorios, que sirvan a las comunidades y grupos de humanos, y que entiendan los medios y las TIC como mediadores y transformadores de la sociedad. 
Estos campos sociales de desempeño profesional, pero también personal, del Comunicador Visual, están marcados por la cuarta revolución industrial, lo que está provocando profundos y revolucionarios cambios en todas las actividades humanas, en especial la social y la económica. La redefinición de paradigmas, cambios y avances tecnológicos permanentes, nuevas relaciones sociales y organizacionales, desmaterialización de los bienes y artefactos, así como nuevos medios y flujos de producción y de trabajo, son algunos de los retos de lo que Bauman denomina "modernidad líquida”, o en términos de Martín Barbero:

"Lo que la trama comunicativa de la revolución tecnológica introduce en nuestras sociedades no es tanto una cantidad inusitada de nuevas máquinas sino un nuevo modo de relación entre los procesos simbólicos -que constituyen lo culturaly las formas de producción y distribución de los bienes y servicios. El nuevo modo de producir, inextricablemente asociado a un nuevo modo de comunicar, convierte al conocimiento en una fuerza productiva directa. La "sociedad de la información” no es entonces sólo aquella en la que la materia prima más costosa es el conocimiento sino también aquella en la que el desarrollo económico, social y político, se hallan estrechamente ligados a la innovación, que es el nuevo nombre de la creatividad y la creación humanas”. (Martín-Barbero, 2020, pág. 8).

Un mundo lleno de conceptos en construcción y redefinición como: inteligencia artificial, blockchain, big data, computación en la nube, cultura digital, cibercultura, ciberciudadanía, ciberactivismo, narrativas expandidas, transmedia e interactivas, internet de las cosas, diseño de experiencia del cliente (CXD), diseño de experiencia del usuario (UXD), Apps vs. Dapps, realidad virtual, aumentada y mixta, slow media y las que aparezcan, implican la necesidad de la disciplina emergente de Comunicador Visual. Esta Profesional tiene el reto y el deber de abordar: múltiples idiomas, lenguajes, medios, técnicas y tecnologías, todos en constante cambio, mutación y actualización. Ser flexible y capaz de adaptarse al cambio.

El Comunicador Visual crea, gestiona y pone en circulación proyectos de comunicación visual éticos, estéticos, estratégicos, creativos y socialmente responsables, adecuados a los contextos donde se presenten dado que son fruto y hacen parte de los bienes culturales y creativos de la sociedad.

De allí que la Comunicación Visual se orienta hacia el desarrollo humano y social integral, el aporte a las comunidades, y la sensibilidad frente a las diversas problemáticas del contexto, aportando la formulación y ejecución de proyectos de comunicación visual como escenarios de creación, significación y representación de la realidad, desde los cuales se desarrollan propuestas situadas y transformadoras de realidades, que permean procesos de construcción social del conocimiento, promueven el respecto por el otro, la ciudadanía participativa, la identidad cultural, la diversidad, la diferencia, el patrimonio y la protección del ambiente.

¿Cuáles son y serán los espacios que ocupe la Comunicación Visual en el marco de la industrias creativas y culturales, más aun en la industria 4.0? ¿Cuáles serán los espacios de formación necesarios a integrar en la formación en Comunicación Visual? 


\section{Referencias bibliográficas}

AIG, G. d. (30 de agosto de 2020). Espacio urbano y tecnologías de género. CARTOGRAFÍA CRÍTICA DEL ARTE Y LA VISUALIDAD EN LA ERA DE LO GLOBAL. Nuevas metodologías, conceptos y enfoques analíticos: https://espaciourbanoytecnologiasgenero.blogs.upv. es/2013/01/23/cartografia-critica-del-arte-y-la-visualidad-en-la-era-de-lo-global-nuevas-metodologias-conceptos-y-enfoques-analiticos/

Ardevol, E., y Muntañola, N. (2014). Representación y cultura audiovisual en la sociedad contemporánea. Editorial UOC.

Brea, J. L. (2003). Estudios visuales. Nota del editor. Estudios visuales No. 1(1), 6.

Buck-Morss, S. (2009). Estudios visuales e imaginación global. Antípoda, 42.

Buitrago, F., y Duque, I. (2013). Economía naranja: una oportunidad infinita. Banco Interamericano de Desarrollo y Puntoaparte Bookvertising.

Costa, J. (2014). Diseño de comunicación visual: el nuevo paradigma, 96.

Estupiñán, A., Solano, A. R., y Torres, M. S. (2014). Lenguaje y significado de la comunicación visual: caso disciplina de comunicación. Actas de Diseño, Vol. 17, (118), 119.

Finkel, M. (8 de Agosto de 2014). Los procesos comunicacionales complejos mediados por las TIC en la comunicación visual mediante la imagen de síntesis en la sociedad del conocimiento. Guatemala, Universidad de San Carlos de Guatemala.

Frascara, J. (2000). Diseño gráfico y comunicación. Ediciones Infinito.

Goyes Narvaez, J. C. (2003). Horizontes de la comunicación visual contemporánea. La Tadeo, No. 68, Primer Semestre, 52.

International Council of Design. (2020, 30 de agosto). ico-D. https://www.ico-d.org/about/ index\#defining-the-profession

Martín-Barbero, J. (2020, 30 de agosto). Organización de los Estados Iberoamericanos. Obtenido de Culturas / Tecnicidades / Comunicación: https://www.oei.es/historico/cultura2/barbero.htm

Max-Neef, M. (1993). Desarrollo a escala humana. Editorial Nordan-Comunidad.

Meggs, P. B. (1991). Historia del diseño gráfico. Trillas. 
Ministerio de Educación Nacional de Colombia. (2003, 30 de diciembre). Resolución número 3436 de 2003. Por la cual se definen las características específicas de calidad para la oferta y desarrollo de los programas de formación profesional en Diseños. Ministerio de Educación Nacional de Colombia.

Moreno, C. (2014). Apuntes de diseño gráfico. Teoría, enseñanza e investigación. Centre d'Etudes Sociales sur Amérique Latine (CESAL).

Pereira, J. M. (2005). La Comunicación: Un campo de conocimiento en construcción. Investigación y Desarrollo. 13(2), 421.

Rollié, R. O., y Branda, M. J. (2004). La enseñanza del diseño de comunicación visual. Editorial Nobuko.

Universidad de Caldas. (2020, 6 de septiembre). Universidad de Caldas. Diseño Visual: https://artesyhumanidades.ucaldas.edu.co/diseno-visual/

Universidad de Medellín. (2020, 30 de agosto). Universidad de Medellín. Comunicación Gráfica Publicitaria: https://udem.edu.co/index.php/2012-10-12-12-56-00/comunicacion-grafica-publicitaria 